\title{
Orthography and the Development of Reading Processes: An Eye-Movement Study of Chinese and English
}

\author{
Gary Feng \\ Duke University
}

\author{
Kevin Miller \\ University of Michigan
}

\author{
Hua Shu and Houcan Zhang \\ Beijing Normal University
}

\begin{abstract}
As children become proficient readers, there are substantial changes in the eye movements that subserve reading. Some of these changes reflect universal developmental factors while others may be specific to a particular writing system. This study attempts to disentangle effects of universal and script-dependent factors by comparing the development of eye movements of English and Chinese speakers. Third-grade (English: mean age $=9.1$ years, $n=23$; Chinese: mean age $=9.4$ years, $n=25$ ), fifth-grade (English: mean age $=11.2$ years, $n=30$; Chinese: mean age $=11.4, n=25$ ), and undergraduate students (English: $n=26$; Chinese: $n=30$ ) read stories in their native language while their eye movements were recorded. Results show a mixture of orthography-dependent factors with others that are remarkably parallel across these two very different writing systems. Orthographic effects are also more pronounced for children than for skilled adult readers. Implications for theories of reading eye movements and reading development are discussed.
\end{abstract}

As children become proficient readers, the mechanics of reading eye movements undergo substantial changes. On average the duration of fixations (the period when the eyes stay relatively motionless) decreases, and the length of saccades (the jumps between fixations) increases. Between them, these changes lead to a more than tripling of reading speed between first and twelfth grade according to one estimate (Taylor, 1965). This is a remarkable change, and it doubtless reflects increased reading expertise and other developmental factors, as well as features of the writing system being read. The goal of the present study was to delineate how these factors influence the moment-by-moment decision of when and where to move one's eyes in reading.

Two broad classes of factors might account for these changes. The first involves universal improvement of efficiency in the course of reading acquisition regardless of the language or writing system. Some of these changes may reflect maturational processes of the brain, for example, the ability

This study was supported by National Institutes of Health Grant 5R03HD051909-02 to the first author and by National Science Foundation Grant BCS 00-79973 to the second author.

Correspondence concerning this article should be addressed to Gary Feng, Department of Psychology \& Neuroscience, P.O. Box 90085, Duke University, Durham, NC 27708. Electronic mail may be sent to garyfeng@duke.edu. to inhibit inappropriate saccadic eye movements (Fischer, Biscaldi, \& Gezeck, 1997; Klein \& Foerster, 2001) or the remarkable increases in processing speed that occur across many cognitive domains (Kail, 1986). In other cases, increasing reading facility involves general factors that are inherent in the process of reading such as word recognition and sentence comprehension. A case in point is the perceptual span in reading, which is the region within which useful visual information is perceived (Rayner, 1998). Rayner (1986) reported that the perceptual span expands with age and reading expertise. These processes are experience related rather than maturational, but they reflect aspects of reading that are so universal that experience in reading any orthography should lead to similar patterns of increasing eye-movement efficiency.

The other class of factors that might account for increasingly efficient eye-movement control with developing reading skill involves efficient adaptation and exploitation of the features of a particular orthography. To the extent that writing systems differ in their structure and fundamental units, one might expect that the developmental course of

(C) 2009, Copyright the Author(s)

Journal Compilation (C) 2009, Society for Research in Child Development, Inc. All rights reserved. 0009-3920/2009/8003-0009 
reading eye-movement control would differ among children learning to read such different orthographies. This would only be true to the extent that children's reading processes are sensitive to the factors represented by those orthographic differences, and so understanding the nature and timing of the effects of orthography on reading will provide insights into the developing flexibility of reading processes.

In an attempt to begin to distinguish contributions of universal and orthography-dependent factors to reading development, the present study compares eye movements of children and adults who are speakers of two very different languages English and Chinese. Because the orthographies used to write these two languages differ so greatly, comparisons of the developmental course of reading in Chinese and English provide a good basis for beginning to disentangle script-dependent aspects of reading development from those that rely on more universal aspects of reading and its acquisition.

Before introducing the design of the study, we will first review existing data on developmental changes in reading eye movements of readers of English, then discuss features of the English and Chinese orthographies relevant to reading, and finally discuss existing eye movement data on reading in these two orthographies.

\section{Eye Movement Control in Reading}

During reading, the eye alternates between jerky movements called saccades and brief stops called fixations. The primary function of eye movements is to bring new words to the retina because what we can perceive during a fixation is limited by the perceptual span, which is typically less than 20 letters for English reading adults (see Rayner, 1998). This is a challenging task. The decision of where to send the eye has to be made very rapidly (typically under $200 \mathrm{~ms}$ ) with limited visual information. And when the move turns out to be nonoptimal, the reader is stuck with the result until the next saccade. In some sense, eye movements are a bottleneck of information processing in reading, that is, they control the speed and order of information intake. Thus, efficient eye movement planning is not only a reflection of reading expertise, but also a critical part of the reading apparatus.

There are two important decisions in reading eye movement planning: (a) where to send the eye and (b) when to terminate the present fixation and move the eye. There is mounting evidence that they are made by two separable but overlapping neural pathways, particularly in the superior colliculus and the brain stem (Findlay \& Walker, 1999). Feng (2006a) also showed that the two pathways can be simulated as separate Markov processes in reading.

This raises the possibility that various aspects of reading eye movements may be differentially sensitive to maturational and experiential changes. There is some suggestive evidence from nonreading oculomotor studies. Fischer et al. (1997) showed that the oculomotor processes for voluntary control of saccades (initiating saccades and inhibiting inappropriate responses) correlate with age, but those for maintaining fixations do not. The former has been linked with prefrontal maturation (Klein \& Foerster, 2001), whereas the latter is mostly a function of the superior colliculus and other visionrelated centers. Voluntary control is involved in choosing the next word target, although it may also play a role in determining the duration of fixations. Thus these results suggest strong developmental changes in the where pathway in reading, although the specific orthography may or may not have an impact on the where pathway. In addition, some aspects of the when decision may be controlled by robust neurological processes that are not influenced by either reading experience or age.

\section{Developmental Changes in Reading Eye Movements}

Much of what we know about reading eye movements comes from studies of English-speaking adults (see Rayner, 1998); studies on the normative development of reading eye movements remain few and far between (see Buswell, 1922; Judd et al., 1918; McConkie et al., 1991; Rayner, 1986; Taylor, 1965). In general, results show that the efficiency of eye movements increases as a function of age and reading proficiency. For example, McConkie et al. (1991) found that the average fixation duration decreased from $304 \mathrm{~ms}$ in first grade to $262 \mathrm{~ms}$ in third grade and then to $243 \mathrm{~ms}$ in fifth grade, and the mean saccade length increased from 3.6 letters to 5.7 and to 6.3 letters, respectively. In addition, children change their scanning patterns. The number of fixations per word drops continuously from first through sixth grade (Buswell, 1922; Rayner, 1986, 1998; Taylor, 1965). This is largely driven by a reduction of within-word refixations and an increase of word skipping (McConkie et al., 1991), both of which are signs of proficient reading.

Developmental studies also hint that some aspects of eye movements may show little change with age. Three examples will be briefly discussed here. The first is the landing position distribution, or where the eye looks within a word (McConkie, 
Kerr, Reddix, \& Zola, 1988). Adult readers generally target the center of the word because word recognition is most efficient there. First-grade students show almost identical landing position distributions to those of skilled readers (McConkie et al., 1991), suggesting that this low-level oculomotor optimization is already in place when children begin to read. Second, the mechanism that controls fixation duration is also less susceptible to developmental forces. Although mean fixation duration declines with age, the mode (peak) of the fixation duration distribution remains nearly constant across age groups, at around $180 \mathrm{~ms}$ (McConkie et al., 1991). Young readers have longer mean fixation durations primarily because of higher proportions of long fixations, which are often associated with cognitive control and inhibitory processes (Yang \& McConkie, 2001). Finally, across a number of studies, the proportion of regressive eye movements has been found to remain relatively flat throughout elementary years (see McConkie et al., 1991); the regression rate varies between $20 \%$ and $33 \%$, depending on the studies. There is currently no convincing explanation for this phenomenon.

In summary, there is converging evidence that aspects of the oculomotor system may show a different developmental course. One question impossible to answer from studying readers of alphabetic scripts is the extent to which the development of eye-movement control processes are affected by features of the writing system children are learning to read. By comparing the development of children who are learning to read very different orthographies, we hope to begin the process of understanding the nature, timing, and limits of influences of orthography on the one hand, and maturation and general reading experience on the other, on the development of eye-movement control processes that subserve skilled reading.

\section{Chinese and English Orthographies: Features Relevant to Reading}

Chinese characters and the alphabetic orthography used to write English differ in a number of features with the potential to affect reading acquisition. The main features relevant here are: (a) the fundamental linguistic unit represented by the orthography, (b) the relative transparency of the orthography, and (c) the orthographic marking of boundaries between units. These features interact to produce important differences in the perceptible information available to readers of these two orthographies.
Fundamental orthographic units. English words are composed of a series of letters, which very roughly correspond to phonemes. This is not to say that English letters correspond to phonemes in any straightforward way (Treiman, Weatherston, \& Berch, 1994). With very rare exceptions, Chinese characters represent morphemes, and correspond to syllables in the spoken language. Characters cannot be further decomposed into smaller phonological units. Thus, the two scripts differ in the phonological transparency, i.e., the likelihood that a reader can pronounce an unfamiliar word.

Transparency of writing systems. Both writing systems encode phonological and morphological information, but with distinct approaches. The type and consistency of information afforded by the orthography determine the efficacy of beginning readers' decoding strategy (Seymour, Aro, \& Erskine, 2003).

The Chinese writing system provides hints to both the sound and meaning of a character, though their usefulness depends on the character and the reader. About $90 \%$ of Chinese characters are semantic-phonetic compounds (Hoosain, 1991), consisting of one part that has some relation to the pronunciation of the character and one part that has some relation to its meaning. One would have a better chance guessing an unfamiliar semanticphonetic compound character than other types of characters. Nevertheless, the proportion of these characters is smaller in early grades; that is, it accounts for $45 \%$ of characters introduced in the first grade compared with $86 \%$ in the fifth grade (Shu, Chen, Anderson, Wu, \& Xuan, 2003). Phonologically consistent characters, characters that had the same pronunciation as their phonetic components or differed only by tone, rose from $32 \%$ in the first grade to $43 \%$ in the sixth grade. Thus, the morphology of Chinese characters provides useful clues to their pronunciation, but not in a way that children can expect to be able to "sound out" a new character. This is particularly the case for young children, who are more likely to be learning characters whose pronunciation cannot be derived from a phonetic component; even if it does, young readers may not know the character that serves as a phonetic component in a new character. There are other ways that characters are formed. For example, a small percentage of characters $(<1 \%$ according to DeFrancis, 1989) have their origin in pictographic representations of a concept. Because these characters have become conventionalized over time, the meaning of such characters is rarely evident to readers. 
As is the case with Chinese, English contains many exception words whose pronunciation cannot be inferred from their written representations (Nagy, Anderson, \& Herman, 1987). But in general, a skilled reader has a good chance of guessing most, if not all, sounds of an unfamiliar English word (Venezky, 1999). The greater likelihood of being able to figure out new words for English may have consequences for eye movement control. For example, readers of English may be more likely to gain new information by repeatedly inspecting (refixating) a word, whereas the same strategy is much less likely to pay off in reading Chinese, particularly for young readers.

Orthographic marking of word boundaries. A major difference between the two orthographic systems is that English texts consist of a series of words separated by spaces, whereas Chinese texts consist of a series of evenly spaced characters that largely correspond to morphemes. Words in modern Chinese consist of one or more characters (with an average of 1.5 characters; Feng, 2006b; Sun, Morita, \& Stark, 1985) but they are not marked in any form in writing. Consequently, there is little consensus among Chinese speakers about what words are (Miller, Chen, \& Zhang, 2007; Wang, 2003).

The difference in word vs. morpheme spacing may have both conceptual and perceptual consequences (Feng, 2008). If words are essential in the comprehension process, one would predict some processing costs associated with assembling characters into words in reading Chinese, and these costs should be larger for beginning readers. The lack of word spacing may also lead to costs in visual perception and oculomotor planning. Skilled readers of English acquire word-length information parafoveally and use it in deciding where to look next (Morris, Rayner, \& Pollatsek, 1990; Rayner, Sereno, \& Raney, 1996). The lack of word boundary demarcations in Chinese would preclude these useful oculomotor strategies and could potentially slow down Chinese reading.

Interestingly, the opposite prediction may also be made. Past research on Chinese reading has shown that adding spaces to separate words resulted in either no effect (Everson, 1986) or negative effects on comprehension (Liu, Yeh, Wang, \& Chang, 1974; see Feng, 2006b, for a review). Thus it may be that skilled readers of Chinese can organize characters (morphemes) into words without taking resources away from reading comprehension. Furthermore, there may be a trade-off between marking word boundaries (as in English) versus morpheme boundaries (as in Chinese). Although word spacing may make saccade programming a relatively straightforward task in English reading, it may impose costs as well as benefits because morphological boundaries are not marked (Reichle \& Perfetti, 2003).

There is some evidence supporting this possibility. Long words are more likely to be refixated, and this may reflect some kind of morphological processing in which words are broken into smaller units. German is a language notorious for its long words (Twain, 1880/1997). Inhoff, Radach, and Heller (2000) showed when spaces are inserted to break long words into smaller, meaningful units, native German readers performed better on naming and reading tasks. Evidence of morphological processing costs has also been reported in Finnish and English compound word reading (Andrews, Miller, \& Rayner, 2004; Bertram \& Hyona, 2003; Hyona, Bertram, \& Pollatsek, 2004). These studies involve skilled readers only; whether and when sensitivity to the unit marked by an orthographic system develops will be explored in this study.

\section{Reading in Chinese and English: Eye Movement Patterns}

Given the large differences between the writing systems, one might predict distinct patterns of eye movements in reading Chinese and English. Research in the last 80 years, however, suggests that there are more similarities than differences among skilled readers of these two orthographies (Feng, 2006b; Feng, Miller, Shu, \& Zhang, 2001; Gray, 1956; Hsien-Ming Yang \& McConkie, 1999; Miles \& Shen, 1925; Peng, Orchard, \& Stern, 1983; Sun et al., 1985; Tsai \& McConkie, 1995, 2003; Yang, 1994; Yang \& McConkie, 1994). For example, when native Chinese or English speakers read comparable scientific articles (Sun \& Feng, 1999), critical eye movement variables, such as mean fixation durations (257 $\mathrm{ms}$ vs. $265 \mathrm{~m}$ ), average saccade length (1.71 vs. 1.75 words per fixation), and reading rate (386 vs. 382 words per minute) were remarkably similar. The perceptual span, the size of effective vision during a fixation (McConkie \& Rayner, 1975) is also comparable among adult readers of Chinese and English when measured in words, not letter spaces (Inhoff \& Liu, 1998; Tsai \& McConkie, 1995; Tsai, Tzeng, Hung, \& Yen, 2000).

Eye-movement studies of Chinese reading are still quite rare. Chen, Song, Lau, Wong, and Tang (2003) reported data involving second-, fourth-, and sixth-grade students from Hong Kong reading sixth-grade-level prose passages. Mean first fixation 
duration was 280, 253, and $232 \mathrm{ms,} \mathrm{respectively,} \mathrm{for}$ the three grades. Average forward saccade length increased from 2.3 character spaces for the second graders to 2.6 for the older grades. The probability of regression remained virtually flat at around $15 \%-17 \%$. A decrease in the rate of within-word refixation was hinted by the large decline in gaze duration (sum of duration of all fixations on a word before the eye leaves the word). Overall, the developmental pattern reported in Chen et al. is broadly consistent with the literature on English reading development summarized before.

Although research looking at college students' reading of Chinese and English has been notable for the cross-language consistency found in the mechanics of reading, this need not imply that the same patterns will be found for children. With less reading experience and more fragile skills, young children may be more affected by the characteristics of orthography than are adults. To our knowledge, the study reported here is the first cross-language developmental study that compares eye movements of Chinese and English speakers.

\section{Predictions}

Studies of the development of reading processes in children learning to read Chinese and English provide a powerful basis for disentangling the role of universal and script-dependent processes in the development of reading skill. Logically, there are three possible patterns of development among the different components of reading eye-movements:

Parallel development across writing systems. To the extent that some aspects of eye-movement control show parallel development across two very different writing systems, developmental explanations that refer to global cognitive changes or universal aspects of the reading process are implicated. This is a plausible hypothesis for at least two reasons. First, maturational processes have been shown to play important roles in oculomotor development (Fischer et al., 1997). Second, existing studies suggest that reading Chinese is remarkably similar to reading English among skilled readers of the two orthographies (e.g., Gray, 1956; Sun \& Feng, 1999). Whether the same pattern holds across development is a question to be addressed in this research.

Script-dependent developmental patterns. As described above, Chinese characters and English alphabetic writing differ profoundly in their visual organization and in the way in which they represent their respective spoken languages. These differences may impose different demands on the oculomotor system and result in fundamentally different reading processes. Specifically, because (a) information is more densely packed in Chinese characters compared with English words (Hoosain, 1991), (b) characters provide less systematic phonological information, and (c) Chinese words are not separated by spaces, one might predict that Chinese children should tend to make shorter saccades and longer fixations on average, compared with their American peers. Together, these should lead to slower reading speeds among Chinese children.

But one might also make the opposite prediction. Chinese-speaking children may have developed script-specific oculomotor strategies early on, and thus not be negatively affected by the lack of word spacing. In addition, they may benefit from not having to break long words into smaller meaning units. If breaking up is indeed hard to do, then it should be readers of English who show disruption because of the need to parse long perceptual units in the course of reading. This disruption should show up in relatively more refixations of words among readers of English compared with their Chinese peers.

Different pathways for different aspects of eye-movement control. The development of reading eye movements may be driven by a combination of script-dependent features and others that reflect maturation or aspects of reading that do not vary across writing systems. Fischer et al. (1997) distinguished between neural systems that control where one looks and when one changes looking position. Based on prior research with children learning to read English, it is possible that these systems might show different patterns of variation across orthographies, with orthography affecting where children look more than how long children look during fixations.

Differential cross-orthography developmental patterns would provide strong support for the differentiation between these two eye-movement control systems. This model leads to the following predictions: Fixation duration should show similar developmental trajectories across age (McConkie et al., 1991) and languages (Sun \& Feng, 1999). Saccade planning, on the other hand, may be more susceptible to cognitive control (such as different reading strategies) as well as to influences of idiosyncratic features of writing systems. We predict that saccade-related measures will demonstrate (a) substantial developmental changes and (b) clear cross-language differences. 


\section{Method}

\section{Participants}

The American participants were 23 third-grade students (mean age $=9.1$ years, range $=8.6-10.2 ; 12$ males and 11 females), 30 fifth-grade students (mean age $=11.2$ years, range $=10.6-12.1 ; 15$ males and 15 females), and 26 undergraduate students (14 males and 12 females) from two small towns in east central Illinois. They were primarily Caucasians. As a group, children from the two Illinois schools tested above the 80th percentile in the Illinois State reading assessments. The Chinese participants were 25 third-grade students (mean age $=9.4$ years, range $=9.0-10.6 ; 12$ males, 13 females), 25 fifthgrade students (mean age $=11.4$, range $=10.7-11.9$; 12 males and 13 females), and 30 undergraduate students (14 males and 16 females) from Beijing, China. The Chinese school is located near downtown Beijing. At the time of the study, it was well equipped but was rated as average in academic performance in its district. All participants in both countries had either normal vision or corrected to normal vision.

\section{Materials}

The selection of reading materials was subject to conflicting constraints. On the one hand, they should be age and culture-specific and reflect materials children might encounter in their daily lives; a one-size-fit-all approach could introduce unwanted age and cultural biases. On the other hand, a common metric is required to compare reading eye movement patterns across languages and age. The compromise was a mixed design (see Appendix A for more details).

To compare developmental changes in reading eye movements on a common metric, a core subset of materials was read by all readers in their own languages. This included two passages "The Power of Boats" (BOAT) and "The Foolish Mule" (MULE) that had parallel English and Chinese versions and were within the reading abilities of both third- and fifth-grade participants. The two stories and the comprehension questions were originally used in a cross-cultural study of American, Japanese, and Chinese students' reading abilities (Stevenson et al., 1990). Cultural biases and difficulty levels of these stories were controlled in the development of these materials. They were rated as having fourth-grade and fifth-grade difficulty levels, respectively, in Stevenson et al.'s (1990) study, but our pretests and teacher interviews showed that most third graders had no difficulty with them. These two stories served as anchors for cross-cultural and cross-age comparisons.

An equally important concern was that reading materials should reflect the cultural value and daily experience of readers, particularly young readers, of the two countries. To this end, different materials were selected for each grade and language. We did this by first obtaining a selection of stories from popular third- and fifth-grade extracurricular reading series in each country. We selected two stories from the third-grade series and two from the fifthgrade series for each country that were roughly matched in terms of their contents (e.g., biography, science, etc.) and approximate length. Teachers of the participating classes read the passages and judged them to be appropriate for their students. As a check on the difficulty of the stories, we asked teachers in both countries to circle words (English) or characters (Chinese), that they thought would be difficult for an "average" student in their classes. On average, the Chinese materials contained slightly more unfamiliar characters (25 for third grade and 6 for fifth grade) compared with unfamiliar words in the English materials (17 for third grade and 7 for fifth grade). These passages provide a sampling of the kinds of reading that children in each country might be naturally exposed to, but none of our participants had read the stories prior to the experiment. We generated two comprehension questions for each story.

Finally, we asked adult readers to read the same stories as young readers did. The adult experiments were not designed to measure how skilled readers read reading-level appropriate materials. The focus of the present study is the nature of children's reading and its development. We included adults in each country primarily to get a baseline measure of how skilled readers would read the kinds of materials we were showing to children. As a result, adult readers' reading and eye movement behaviors may differ from when they read more sophisticated materials.

Reading materials were presented on a computer screen $60-70 \mathrm{~cm}$ away from the reader. Children read the stories selected for their age group; adults read all of the stories. The Chinese materials were displayed in $24 \times 24$ pixel Song font, corresponding to about 1.3 visual degrees per character with 4 pixels space between characters. An English letter was on average 7.3 pixels wide or 0.35 visual degrees. Each screen held a maximum of seven lines of English text (45 pixels between lines) or six lines of Chinese text (48 pixels between lines). The 
line spaces were adequate to allow for typical vertical drifts of the Eyelink eye tracker.

Font sizes and line spaces were chosen to facilitate comparison of eye movement parameters between the two languages. Cross-linguistic comparisons of saccade length are complicated by the fact that there is not a common metric for English and Chinese texts. Because reading saccades are primarily affected by linguistic units (number of letters or words) rather than by font size and viewing distance (Rayner, 1998), saccade length is typically reported in letter spaces for English reading but in character spaces for Chinese reading. Arbitrary scaling factors have been proposed, such as two English letters for a Chinese character (Yang \& McConkie, 1994), but their appropriateness is questionable (e.g., Feng, 2006b; Hoosain, 1991). In the present study, we adjusted the font size in both languages so that a parallel story in English and Chinese (i.e., the MULE story) would have approximately the same number of lines in both languages. As a result, a Chinese character equals 3.9 English letter spaces. A typical six-letter English word therefore took approximately the same space as the average length of a Chinese word, which is 1.5 characters (Hoosain, 1991; Tsai, Lee, Hung, \& Tzeng, 2001). This was obviously a crude attempt to equate the materials and was never intended to be a precise matching. Nevertheless, it allowed a comparison of saccade length in term of the screen pixels. Although any cross-language comparison of saccade-length will be problematic, developmental changes within country do not have these concerns.

As noted previously, the Chinese orthography does not mark word boundaries, and educated Chinese-speaking adults do not necessarily agree on how to divide a passage into words (Hoosain, 1991; Miller et al., 2007; Wang, 2003). We identified words based on a word-segmentation protocol known as the Chinese National Standard GB13715 (Liu, Tan, \& Shen, 1993). Statistics and analyzes involving word units in Chinese are all based on this standard.

\section{Apparatus and Procedure}

Participants were asked to read the stories silently as they ordinarily would and were informed that they would be asked some questions about each story after they completed reading it. Readers' eye movements were recorded with an EyeLink I eye-tracking system, which is a head-mounted infrared system with $250-\mathrm{Hz}$ sampling rate and a maximum spatial resolution of $0.005^{\circ}$, although the typical accuracy level is approximately 0.5 visual degree, measured by repeated calibrations. Saccade detection was based on a built-in acceleration-based algorithm with default parameters for reading and cognitive studies. No head support or chin rest was used. Head movements were corrected by the builtin algorithm in the eye tracker.

The English and Chinese studies were both run by the first author and the procedure was identical. A 9-point calibration was performed before each experiment and was repeated as necessary during the experiment. A calibration was accepted when average error over the 9 points was less than 0.8 degrees. A 1-point drift correction was performed between each page. Comprehension questions were presented after each story and were answered orally. The stories were presented in a fixed order in each language. Reading typically took approximately 10-20 min and participants could ask for a break at any time, which several children did but not the adults.

\section{Results}

\section{Reading Comprehension}

The rates of correct answers to the comprehension questions were on average $67.8 \%, 89.2 \%$, and 97.2\% for American third graders, fifth graders, and undergraduate students, respectively, and were $78.0 \%, 81.8 \%$, and $94.4 \%$ for Chinese third graders, fifth graders, and undergraduate students, respectively. Because the reading passages and comprehension questions were different in the two languages, these numbers cannot be compared directly. Comparing comprehension levels for the BOAT and the MULE stories is more straightforward, because the stories and questions are comparable across languages. The mean score for the four questions on these two stories showed no significant country difference between Chinese and American readers, $F(1,163)=3.304, p=.071$, but a significant developmental trend, $F(2,163)=11.619$, $p<.001,(69.3 \%, 83.1 \%$, and $90.4 \%$, for American third graders, fifth graders, and adults; $78.0 \%$, $88.0 \%$, and $97.7 \%$ for Chinese third graders, fifth graders, and adults, respectively).

\section{Reading Speed}

One prediction based on differences between the English and Chinese writing systems is that there may be more processing costs associated with reading Chinese, particularly for Chinese 


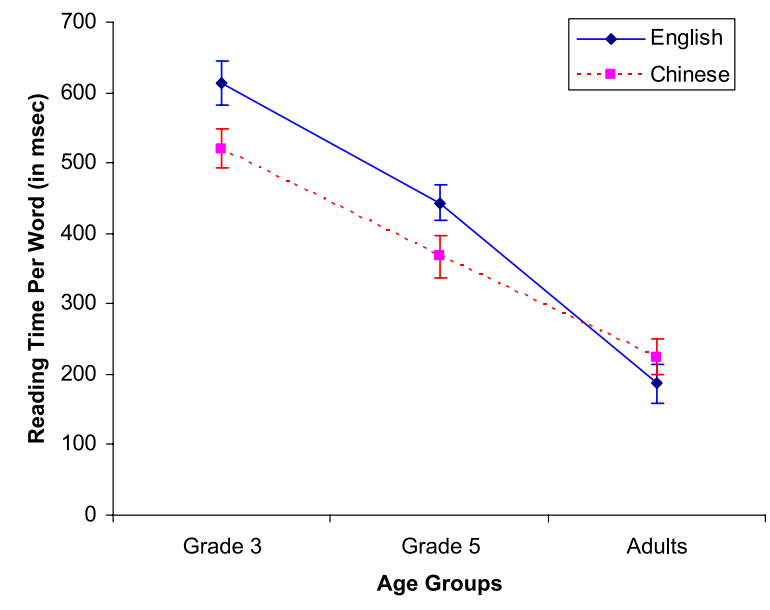

Figure 1. Mean reading time per word.

children. If this is true, one would expect slower reading and longer processing time per word. Figure 1 shows the average reading time per word, averaged across all stories; a separate analysis with only the BOAT and the MULE stories showed the same pattern and therefore is not reported separately. Reading time per word decreased with age, $F(2,148)=83.46 .14, p<.001$, but there was a significant interaction between age and language, $F(2$, $148)=3.36, p=.037$. Paired comparisons within each age group showed that Chinese children read significantly or marginally significantly faster than their American counterparts, $F(1,148)=4.95$, $p=.028$ in third grade, $F(1,148)=3.85, p=.051$ in fifth grade, but the reading time for American and Chinese undergraduate students did not differ significantly, $F(1,148)<1$. American readers' reading speeds, when converted into words per minute (wpm), were approximately 110, 140, and 340 wpm for third grade, fifth grade, and undergraduate students, respectively. The figures for young readers are consistent with figures reported in prior literature. At $340 \mathrm{wpm}$, the reading speed of adult English speakers was faster than averages reported in the literature, approximately $300 \mathrm{wpm}$ (Carver, 1990; Rayner, 1998). However, as Carver (1990) pointed out, a direct comparison of the reading rate may be misleading when materials differ in word length. The average word length in this study (4.03 letters per word) is much shorter than that in adult materials (typically six letters per word; Carver, 1990), which is likely to have inflated the reading rate. Other indicators such as the level of comprehension suggest that adult readers were engaged in meaningful reading behaviors. We were unable to find reliable norms of reading rate in Chinese for either children or skilled readers.

As we expected, although English and Chinese speaking adults read at a similar speed, large language differences were found among beginning readers. The direction of the differences, however, is opposite to the prediction laid out earlier, with an advantage for young Chinese readers despite the lack of explicitly marked word boundaries in their orthography. In the following analyses, we decompose story reading time into two factors-number of fixations per word and mean fixation duration-and explore their relation to reading speed.

\section{Number of Fixations}

The average number of fixations per word was 2.00, 1.56, and 0.79 for American third graders, fifth graders, and adults, and 1.71, 1.31, and 0.86 for their Chinese counterparts, respectively. As shown in Figure 2, the number of fixations per word decreased with age, $F(2,148)=94.30$, $p<.001$, and was lower for Chinese than for English readers, $F(1,148)=6.61, p=.011$. There was again a significant interaction between age and language, $F(2,148)=3.68, p=.028$. The differences were significant at third grade, $F(1,148)=6.52$, $p=.012$, and at fifth grade, $F(1,148)=6.02$, $p=.015$, but nonsignificant for adult English and Chinese readers, $F(1,148)<1$. The same pattern was found when the BOAT and MULE stories were analyzed separately.

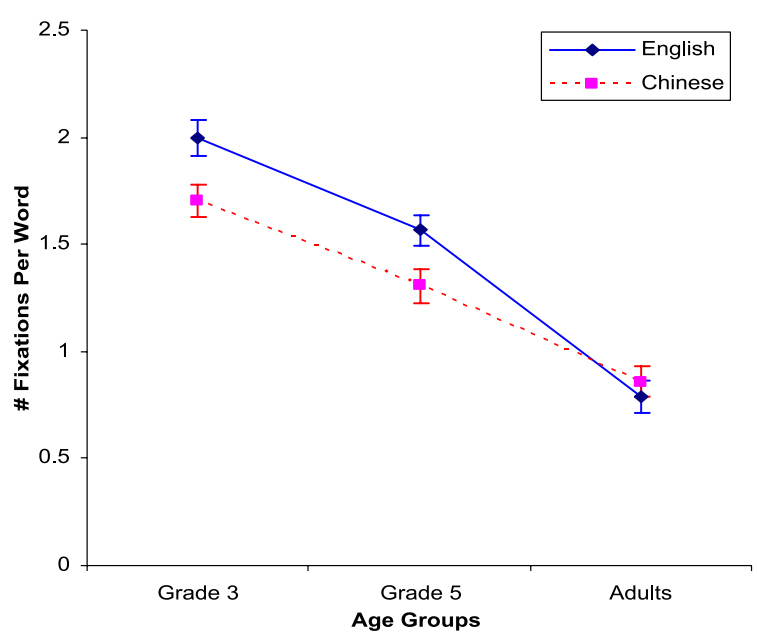

Figure 2. Number of fixations per word. 


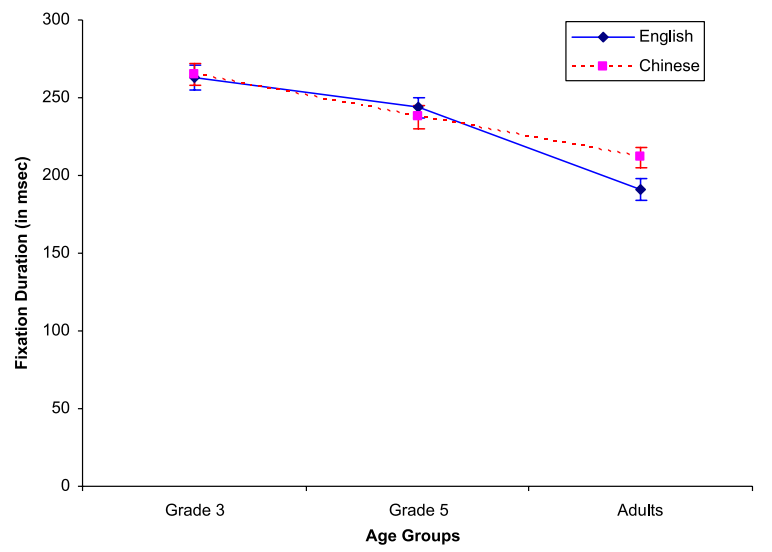

Figure 3. Mean fixation duration for American and Chinese readers.

\section{Fixation Duration}

Mean fixation duration was approximately 263, 244, and $191 \mathrm{~ms}$ for American third-grade, fifthgrade, and adult readers, and 265, 238, and $212 \mathrm{~ms}$ for Chinese third-grade, fifth-grade, and adult readers, respectively. Figure 3 shows the average duration of fixations of each group of participants. Mean fixation duration decreased significantly with age, $F(2,148)=41.27, p<.001$. There was no significant language main effect, $F(1,148)<1$, nor any interaction between age and language, $F(2,148)=2.06, p=.131$. Average fixation duration did not differ significantly between American and Chinese children, $F_{\mathrm{s}}(1$, $148)<1$, for both third and fifth grade, whereas American college students' fixation duration was about $21 \mathrm{~ms}$ faster than that of Chinese college students, $F(1,148)=4.89, p=.029$.

Figure 4 compares the frequency distributions of fixation duration for different reader groups. Only forward fixations were plotted, as regressive fixations are thought to have different functions than acquiring new information (Rayner, 1998). Three features are noteworthy in Figure 4. First, there is minimal developmental or cross-cultural difference in the peaks of the distributions. All fixation duration distributions peak around $180 \mathrm{~ms}$, despite differences in the means. Second, children in both languages differ from adult readers primarily in the right tail of the distribution, that is, children produce higher proportions of long fixations and correspondingly lower percentages of short fixations than adults. Third, this phenomenon (more long fixations among children than adults) is noticeably more pronounced among U.S. than Chinese children. Together, these findings

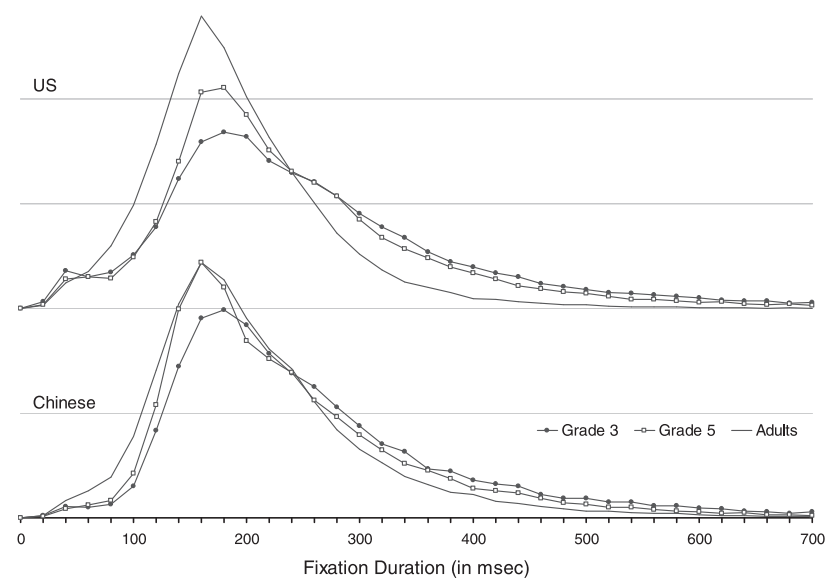

Figure 4. Frequency distributions of fixation durations.

reinforce the conclusion of the relative lack of cultural differences in fixation duration reported earlier. They also add important qualifications to the pattern of mean fixation duration. As children become more proficient in reading, there is a reduction in very long fixations, a phenomenon more pronounced among those learning to read English.

\section{Distributions of Saccade Length}

As mentioned previously, comparing eye movement parameters is complicated by the different unit of writing in English and Chinese. Our approach, which was far from the only one possible, was to try to ensure texts of the same story occupy approximately the same linear space, in terms of pixels. Thus, the stories take up the same horizontal space in total, and analyzes of saccades can be based on pixels. We will also report a word-based analysis of saccades. Because the texts are of the same size, effects of the different orthographies would show up in different patterns of distributions of saccades among forward fixations, regressions, and within-word refixations.

On average, American readers' forward saccades were significantly shorter than those of Chinese readers. The length of forward saccades increased with age, $F(2,148)=81.82, p<.001$, and was longer for Chinese than for English readers, $F(1$, $148)=53.24, p<.001$, and there was no interaction between age and language, $F(2,148)<1$. There were significant country differences for each age group: $F(1,148)=13.41, p<.001$ at third grade; $F(1,148)=24.53, \quad p<.001$ at fifth grade; and 


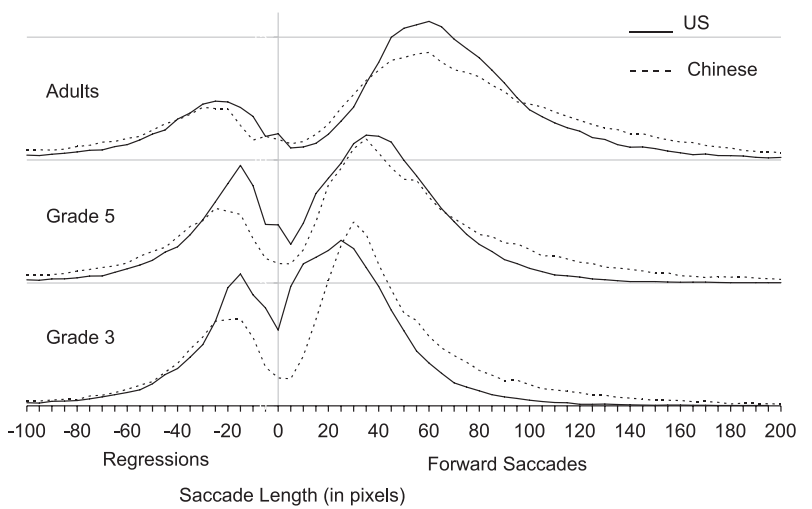

Figure 5. Frequency distributions of saccade length.

$F(1,148)=16.64, p<.001$ for adults. Mean saccade lengths for American readers were approximately 5.5, 6.7, and 10.3 letter spaces for third-grade, fifthgrade, and adult readers, respectively. Saccade length averaged 2, 2.4, and 3.1 characters for Chinese third-grade, fifth-grade, and undergraduate students, respectively.

Frequency distributions of forward saccade length (in pixels) for American and Chinese children and adults are shown in Figure 5. The peaks of English and Chinese adult readers coincide, possibly because of our effort to equate the physical length. But the distributions differ otherwise, with American children making more short saccades and fewer long saccades than their Chinese peers. Because a typical five-letter English word is approximately 37 pixels long, many of the short saccades made by American children would land on the same words. American undergraduate students, while still making fewer long saccades, were no more likely to make these short saccades than the Chinese counterparts.
Kinds of Saccades: Forward, Refixations, and Regressions

The large number of short saccades in American children's saccade distributions suggests that they made more within-word refixations. To test this hypothesis, we divided fixations into three categories-progressive fixations, interword regressions, and refixations-based on whether they landed on a new word, a word that had been read, or the current word, respectively. The three categories are defined based on words, for example, a leftward saccade that landed on the same word-not infrequent in reading-counts as a refixation. Note that Chinese data were also analyzed by words, as defined in the National Standard GB13715, rather than characters in this analysis. The percentages of each category within each group are shown in Figures 6A-C. As is apparent from the figures, different patterns of country and developmental effects were found for the different kinds of saccades.

Progressive fixations. Progressive fixations increased significantly with age, $F(2,148)=166.79$, $p<.001$, and were higher for Chinese than for English readers, $F(1,148)=10.70, p=.001$, and there was a significant interaction between age and language, $F(2,148)=6.49, p=.002$. The proportions of forward saccades did not differ in adult readers, $F(1,148)=1.06, p=.305$, but differed significantly across languages at third grade, $F(1,148)=11.16$, $p=.001$, and fifth grade, $F(1,148)=9.88, p=.002$. Thus the difference in reading speed reflects the greater proportion of saccades among young Chinese readers that moved forward in the text.

Regressive fixations. A different pattern was found for regressions. There was a significant country effect $F(1.148)=17.67, p<.001$, but not significant age difference nor a significant interaction. At
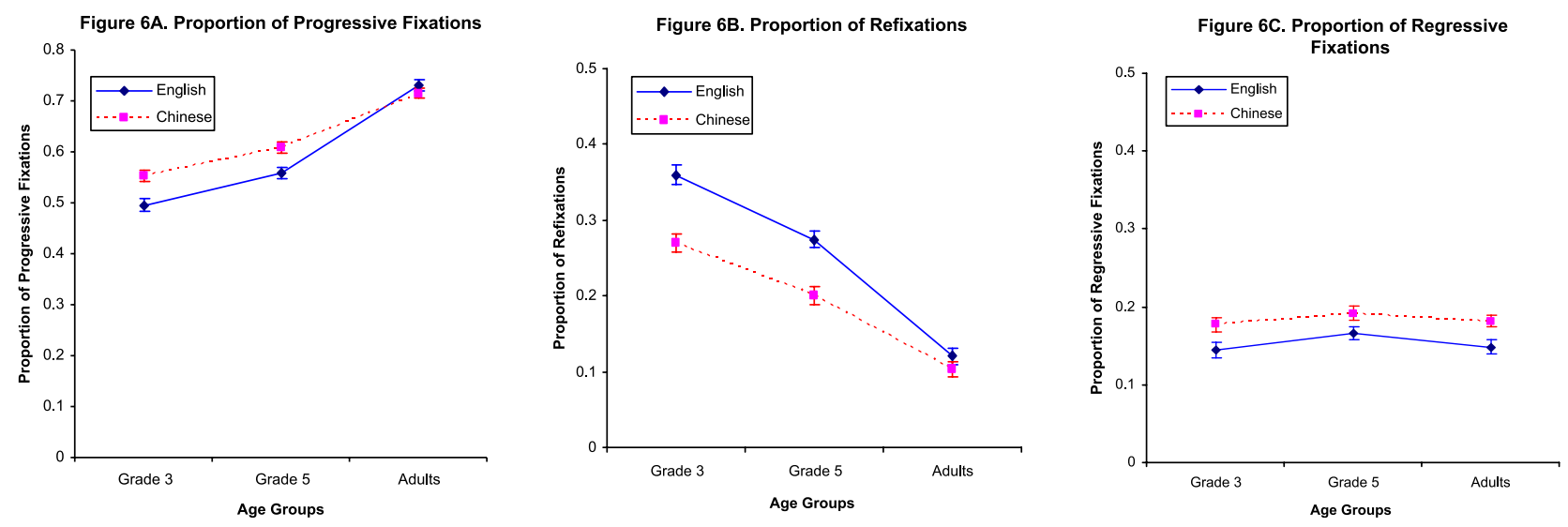

Figure 6. Proportions of progressive fixations, regressive fixations, and refixations. 
all ages, Chinese readers showed more regressions than did American participants, $F(1,148)=6.126$, $p=.014$ for third grade; $F(1,148)=4.12, p=.044$ for fifth grade; and $F(1,148)=7.79, p=.006$ for adults. However, the magnitude of the effect is relatively small compared with other differences in Figure 6. Given that the physical lengths of regressive saccades are similar between the two languages (see Figure 5), we suspect that the crosslinguistic difference may be related to the National Standard GB13715, which tends to aggressively break words into smaller units. For the same saccade length, smaller words will entail higher proportion of between-word regressions. Until a better, psychologically more intuitive word parser is available for Chinese, the present result of language differences in regression rate should be taken with a grain of salt.

We do not have a ready explanation for the lack of developmental changes. However, we are not alone in finding this pattern. Data summarized in McConkie et al. (1991) showed that regression rate remains flat throughout elementary grades; Chen et al. (2003) also found that the regression rate remained at about $15 \%$ among Chinese readers ranging from second grade to college students. The causes of regression in ordinary reading and its relation to reading skill remain unclear, both from this study and from prior research.

Within-word refixations. Refixations decreased with age, $F(2,148)=161.81, p<.001$. There was also a significant language effect, $F(1,148)=41.97$, $p<.001$, and a significant Age $\times$ Language interaction, $F(2,148)=5.67, p=.004$. American children, but not American undergraduate students, made significantly more refixations than their Chinese peers, $F(1,148)=27.14, p<.001$ for third grade; $F(1,148)=21.82, p<.001$ for fifth grade; and $F(1$, $148)=1.31, p=.253$ for adults.

There are at least two plausible explanations for the greater incidence of refixations among young readers of English compared with children learning to read Chinese. The first involves the possibility of sounding-out words in English versus Chinese, and the second involves the problem of parsing English words into smaller morphologically meaningful units. A strategy of refixating unfamiliar words as part of an explicit "sounding out" strategy is certain to be more fruitful in English than in Chinese. This explanation is supported by that fact that we did not find significant differences in refixations between our samples of college students, who should not need to sound out words in the children's texts used in this study.

Because Chinese and English differ in the size of the fundamental unit of writing, the differences in within-word refixations may also reflect problems in parsing words into morphologically meaningful units. Supporting this explanation are the results of Inhoff et al. (2000) who found that segmentation of long German words into smaller units facilitated some (but not all) aspects of reading, even though these smaller words were unfamiliar to their German college student sample. Because we chose to focus on normal text, where word length, word frequency, and regularity are highly confounded (Kliegl, Olson, \& Davidson, 1982), the present study cannot distinguish between these two explanations. Testing these hypotheses will require specially designed materials in carefully controlled contexts. It is, indeed, possible that both factors play a role.

The different patterns of developmental trajectories and cross-language differences for these different components of eye movement control do provide good evidence of the real but limited ways in which the eye movement systems tune themselves to the features of particular orthographies.

\section{Discussion}

To our knowledge, this is the first developmental study that directly compares reading eye movements of English- and Chinese-speaking children. We went to great lengths to ensure the internal and external validity of this cross-linguistic, cross-cultural development study. This is reflected in our sampling decisions, material construction, and measures taken to ensure consistency and comparability of the data collected from the two very different cultures. The present study focuses on normative development in reading naturalistic texts; this design does not afford testing certain interesting hypotheses on linguistic processing or exploring individual differences in great depth. Adult eye movement parameters also reflect the easy reading materials. Limitations notwithstanding, we believe that this data set allows us to ask a number of important questions, and in some cases, answer them with confidence.

In what follows, we will first compare our results with published data, and summarize the development of reading eye movements in the context of two very different writing systems. We end with an outline of a model to account for the diverse 
developmental and cross-linguistic findings reported here.

\section{Cross-Linguistic Validity}

One of the major challenges for any cross-cultural studies is to ensure the representativeness of the sample of materials and participants. We have several reasons to believe that we were reasonably close to this goal.

Our English language data are in line with previous eye movement studies. For example, the mean fixation duration for American third- and fifth-grade students were 263 and $244 \mathrm{~ms}$, respectively. These figures were almost identical to those reported in McConkie et al. (1991), which were 262 and $243 \mathrm{~ms}$, respectively. Similarly, the average saccade length for third- and fifth-grade students was 5.5, and 6.7 letters in the present study versus 5.7 and 6.3 letters in McConkie et al. (1991), respectively. Basic parameters for English-speaking adult eye movements fall in the typical range, although the slightly shorter mean fixation duration (191 ms) and longer saccade length (10.3 letters) reflected the fact that these stories, which were intended for third- and fifth-grade students, were very easy for adult readers. Chinese adults' eye movement parameters also align well with existing data (see Feng, 2006b). Although our samples of Chinese-speaking children differ in grades from those in Chen et al. (2003) study (Grades 3 and 5 in our study vs. Grades 2, 4, and 6 in Chen et al., 2003), mean fixation durations from the two studies fall almost in a straight line. Average saccade lengths show similar developmental patterns between the two studies. These results, along with the good performance in reading comprehension questions, suggest that our samples-both in terms of participants and texts-are representative of respective age levels within the languages.

\section{Development of Reading Eye Movements}

We hypothesized three possible developmental paths for reading eye movements. First, eye movements may become more efficient because of improvements in overall speed of processing or in general reading-related processes. Correspondingly, there would be no differences in eye movements in reading different orthographies. Alternatively, the mechanism that controls reading eye movements may be orthography specific, particularly for beginning readers who are yet to be freed from effortful word recognition processes. If this is the case, we should observe distinct eye movement patterns among young readers of English and Chinese. Finally, different components of the eye movement mechanism may follow distinct developmental patterns, some of which are language-specific and others language-universal.

Results from the present study provide unequivocal support for the last view. Clearly, different eye movement parameters follow different developmental trajectories; three patterns emerge from the data:

1. Various saccade-related measures continue to develop throughout elementary school, and show considerable differences between English- and Chinese-speaking readers. Consistent with previous research (Fischer et al., 1997; Klein \& Foerster, 2001), this suggests that voluntary control in the where system is linked to the development of cognitive processes-in this case increasingly proficient reading processes. Consequently, differences in saccade patterns reflect different perceptual and cognitive demands Chinese and English reading imposes on the reader. This will be further elaborated in the following section.

2. Beginning readers make higher proportions of long fixations; this pattern is invariant across the two languages and orthographies, suggesting a general developmental trend. Although the present study cannot rule out the possibility that this is due to maturation, a recent theory sheds light on the nature of the phenomenon. In their competition-inhibition theory, Yang and colleagues (Yang, 2006; Yang \& McConkie, 2001) proposed that fixation duration during reading is determined by the competition between two brain centers (the fixate center and the move center). In most cases, fixation duration is determined autonomously by the interaction between these two centers without interference from higher order processes. But when necessary, for example, when comprehension difficulties occur, the cognitive process may intervene by changing the interaction between the two centers and thus extend the current fixation. Assuming beginning readers encounter more difficulties in reading, the competition-inhibition theory predicts more cognitive intervention and thus a higher proportion of long fixations. To the extent that the above assumption is true in both English and Chinese reading, the theory also nicely accounts for the lack of 
language differences in the developmental pattern of fixation durations.

3. Distributions of fixation duration reveal that, with the exception of long fixations discussed earlier, the basic mechanism for fixation control appears to be stable across age and language. This finding corroborates observations from fixation duration distributions in McConkie et al. (1991). It is also consistent with Fischer et al.'s (1997) conclusion that the neurological mechanism that controls fixation duration (the when system) develops early and is mature by school age. Thus, evidence from this and other studies suggests that: (a) the development of fixation duration control is primarily a maturational process that is accomplished by the time children learn to read, and (b) experiential factors (such as reading experience or orthography) have limited influence on this process.

The above conclusion may appear to be in conflict with the notion that fixation duration reflects moment-by-moment cognitive processes in reading (Rayner, 1998; Reichle, Pollatsek, Fisher, \& Rayner, 1998; Reichle, Rayner, \& Pollatsek, 2003). Two clarifications help to resolve the paradox. First, our findings on the rate of refixations and regressions support the notion that cognitive processes affect eye movement planning in real time. The data also suggest that the cognitive influence may be limited to some fixations, leaving other fixations controlled by autonomous oculomotor processes (cf. Yang \& McConkie, 2001; Yang, 2006).

\section{Toward a Theory of Reading Eye Movement Development}

The existence of three distinct development trajectories in reading eye movements calls for a theory of the development of reading eye movement control. Findings from the present study rule out the simplistic notion that all aspects of reading eye movements continue to improve with age and reading experience. In the remainder of this article, we will provide an initial sketch of a model of reading eye movement development that is consistent with observations from this and prior studies (e.g., McConkie et al., 1991).

We speculate that a beginning reader is equipped with the basic oculomotor abilities to maintain fixations and to plan and execute saccades to selected targets. This seems to be a safe assump- tion given that a typical 6-year-old should already have made well over 120 million eye movements (assuming 2 eye movements per second and an average of $8 \mathrm{hr}$ of wakeful time per day). This is also consistent with the finding of McConkie et al. (1991) that first-grade students follow the same oculomotor strategies in targeting words as adult readers. The present study and Fischer et al. (1997) provide evidence that basic fixation duration control is mature by around the time children start to read, if not earlier.

In addition, children have some cognitive control over these oculomotor functions, although the extent to which cognitive processes can overwrite the default oculomotor programs vary with age, the oculomotor function to be controlled, and the nature of the cognitive processes involved. With regard to age, Fischer et al. (1997) found that adults are faster and better at inhibiting reflexive oculomotor programming; this should extend naturally to reading. Furthermore, both Fischer et al. and our data suggest that the when system that controls fixation duration is less susceptible to cognitive control than the where system that is responsible for determining saccade targets. Thus, larger developmental and orthographic differences are observed in saccade-related measures than in fixation duration. This distinction is important for understanding reading development and the underlying neuropsychological mechanisms.

Finally, the driving force behind most developmental changes in reading eye movements is likely to be changes in reading processes that reflect improved reading proficiency. Because processes such as word recognition and text comprehension are highly efficient and automated among skilled readers, there is less need for higher level processes to override default oculomotor programs, which provides an explanation for the remarkable similarities in the eye movements of skilled English and Chinese readers.

Our results support the strategy of looking at young readers to understand the effects of orthography on the cognitive and perceptual mechanisms underlying reading. Reading is a complex process that involves the interaction of a host of perceptual, linguistic, and cognitive factors. Anyone who has read this article to the end surely qualifies as an expert in the coordination of these processes. This very expertise makes disentangling the components of reading difficult. Children, on the other hand, are likely to be much more dependent on and affected by the vagaries of the orthography they are learning to read. 


\section{References}

Andrews, S., Miller, B., \& Rayner, K. (2004). Eye movements and morphological segmentation of compound words: There is a mouse in mousetrap. European Journal of Cognitive Psychology, 16, 285-311.

Bertram, R., \& Hyona, J. (2003). The length of a complex word modifies the role of morphological structure: Evidence from eye movements when reading short and long Finnish compounds. Journal of Memory and Language, 48, 615-634.

Buswell, G. T. (1922). Fundamental reading habits: A study of their development (Vol. 21). Chicago: University of Chicago Press.

Carver, R. P. (1990). Reading rate: A review of research and theory. San Diego, CA: Academic Press.

Chen, H.-C., Song, H., Lau, W. Y., Wong, K. F. E., \& Tang, S. L. (2003). Developmental characteristics of eye movements in reading chinese. In C. McBride-Chang \& H.-C. Chen (Eds.), Reading development in Chinese children (pp. 157-170). Westport, CT: Praeger.

DeFrancis, J. (1989). Visible speech: The diverse oneness of writing systems. Honolulu: University of Hawaii.

Everson, M. E. (1986). The effect of word-unit spacing upon the reading strategies of native and non-native readers of Chinese: An eye-tracking study. Unpublished Ph.D. dissertation, Ohio State University, Columbus.

Feng, G. (2006a). Eye movements as time-series random variables: A stochastic model of eye movement control in reading. Cognitive Systems Research, 7, 70-95.

Feng, G. (2006b). Eye movements in Chinese reading. In P. Li, L. Tan, E. Bates, \& O. J. L. Tzeng (Eds.), Handbook for East Asian psycholinguistics, (Vol. 1, pp. 187-194). Cambridge, UK: Cambridge University Press.

Feng, G. (2008). Orthography and eye movements: The paraorthographic linkage hypothesis. In K. Rayner, D. Shen, X. Bai, \& G. Yan (Eds.), Cognitive and cultural influences on eye movements (pp. 395-420). Tianjin, China: Tianjin People's Press/Psychology Press.

Feng, G., Miller, K., Shu, H., \& Zhang, H. (2001). Rowed to recovery: The use of phonological and orthographic information in reading Chinese and English. Journal of Experimental Psychology: Learning, Memory, \& Cognition, 27, 1079-1100.

Findlay, J. M., \& Walker, R. (1999). A model of saccade generation based on parallel processing and competitive inhibition. Behavioral \& Brain Sciences, 22, 661-721.

Fischer, B., Biscaldi, M., \& Gezeck, S. (1997). On the development of voluntary and reflexive components in human saccade generation. Brain Research, 754, 285-297.

Gray, W. S. (1956). The teaching of reading and writing; an international survey. Paris: UNESCO.

Hoosain, R. (1991). Psycholinguistic implications for linguistic relativity: A case study of Chinese. Mahwah, NJ: Erlbaum.

Hyona, J., Bertram, R., \& Pollatsek, A. (2004). Are long compound words identified serially via their constituents? Evidence from an eye-movement-contingent display change study. Memory and Cognition, 32, 523532.

Inhoff, A. W., \& Liu, W. (1998). The perceptual span and oculomotor activity during the reading of Chinese sentences. Journal of Experimental Psychology: Human Perception and Performance, 24, 20-34.

Inhoff, A. W., Radach, R., \& Heller, D. (2000). Complex compounds in German: Interword spaces facilitate segmentation but hinder assignment of meaning. Journal of Memory and Language, 42, 23-50.

Judd, C. H., Gray, W. S., Gray, C. T., McLaughlin, K., Schmitt, C., \& Gilliland, A. R. (1918). Reading: Its nature and development. Chicago: University Chicago Press.

Kail, R. (1986). Sources of age differences in speed of processing. Child Development, 57, 969-987.

Klein, C., \& Foerster, F. (2001). Development of prosaccade and antisaccade task performance in participants aged 6 to 26 years. Psychophysiology, 38, 179-189.

Kliegl, R. M., Olson, R. K., \& Davidson, B. J. (1982). Regression analyses as a tool for studying reading processes: Comment on Just and Carpenter's eye fixation theory. Memory \& Cognition, 10, 1287-1296.

Liu, Y., Tan, Q., \& Shen, X. (1993). Xinxi Chuli Yong Xiandai Hanyu Fenci Guifan [Modern Chinese word segmentation rules for information processing purposes]. Beijing, China: China Standard Press.

Liu, I. M., Yeh, J. S., Wang, L. H., \& Chang, Y. K. (1974). Effects of arranging Chinese words as units on reading efficiency. Acta Psychologica Taiwanica, 16, 25-32.

McConkie, G. W., Kerr, P. W., Reddix, M. D., \& Zola, D. (1988). Eye movement control during reading: I. The location of initial eye fixations on words. Vision Research, 28, IN1-IN2.

McConkie, G. W., \& Rayner, K. (1975). The span of the effective stimulus during a fixation in reading. Perception $\mathcal{E}$ Psychophysics, 17, 578-586.

McConkie, G. W., Zola, D., Grimes, J., Kerr, P. W., Bryant, N. R., \& Wolff, P. M. (1991). Children's eye movements during reading. In J. F. Stein (Ed.), Vision and visual dyslexia (pp. 251-262). London: Macmillan.

Miles, W. R., \& Shen, E. (1925). Photographic recording of eye movements in the reading of Chinese in vertical and horizontal axes: Method and preliminary results. Journal of Experimental Psychology, 8, 344-362.

Miller, K. F., Chen, S.-Y., \& Zhang, S. (2007). Where the words are: Judgments of words, phrases and syllables by speakers of Chinese and English. Unpublished manuscript.

Morris, R. K., Rayner, K., \& Pollatsek, A. (1990). Eye movement guidance in reading: The role of parafoveal letter and space information. Journal of Experimental Psychology: Human Perception and Performance, 16, 268281.

Nagy, W. E., Anderson, R. C., \& Herman, P. A. (1987). Learning word meanings from context during normal reading. American Educational Research Journal, 24, 237270.

Peng, D. L., Orchard, L. N., \& Stern, J. A. (1983). Evaluation of eye movement variables of Chinese and 
American readers. Pavlovian Journal of Biological Science, 18, 94-102.

Rayner, K. (1986). Eye movements and the perceptual span in beginning and skilled readers. Journal of Experimental Child Psychology, 41, 211-236.

Rayner, K. (1998). Eye movements in reading and information processing: 20 years of research. Psychological Bulletin, 124, 372-422.

Rayner, K., Sereno, S. C., \& Raney, G. E. (1996). Eye movement control in reading: A comparison of two types of models. Journal of Experimental Psychology: Human Perception and Performance, 22, 11881200.

Reichle, E. D., \& Perfetti, C. A. (2003). Morphology in word identification: A word-experience model that accounts for morpheme frequency effects. Scientific Studies of Reading, 7, 219-237.

Reichle, E. D., Pollatsek, A., Fisher, D. L., \& Rayner, K. (1998). Toward a model of eye movement control in reading. Psychological Review, 105, 125-157.

Reichle, E. D., Rayner, K., \& Pollatsek, A. (2003). The E-Z Reader model of eye-movement control in reading: Comparisons to other models. Behavioral and Brain Sciences, 26, 445-476.

Seymour, P. H. K., Aro, M., \& Erskine, J. M. (2003). Foundation literacy acquisition in European orthographies. British Journal of Psychology, 94, 143-174.

Shu, H., Chen, X., Anderson, R. C., Wu, N., \& Xuan, Y. (2003). Properties of school Chinese: Implications for learning to read. Child Development, 74, 27-47.

Stevenson, H. W., Lee, S.-y., Chen, C., Stigler, J. W., Hsu, C. C., \& Kitamura, S. (1990). Contexts of achievement: A study of American, Chinese, and Japanese children. Monographs of the Society for Research in Child Development, 55.

Sun, F., \& Feng, D. (1999). Eye movements in reading Chinese and English text. In J. Wang, A. W. Inhoff, \& H.-C. Chen (Eds.), Reading Chinese script: A cognitive analysis (pp. 189-206). Mahwah, NJ: Erlbaum.

Sun, F., Morita, M., \& Stark, L. W. (1985). Comparative patterns of reading eye movement in Chinese and English. Perception \& Psychophysics, 37, 502-506.

Taylor, S. E. (1965). Eye movements in reading: Facts and fallacies. American Educational Research Journal, 2, 187202.

Treiman, R., Weatherston, S., \& Berch, D. (1994). The role of letter names in children's learning of phoneme- grapheme relations. Applied Psycholinguistics, 15, 97122.

Tsai, J. L., Lee, C. Y., Hung, D. L., \& Tzeng, O. J. L. (2001, August). Eye movement guidance without word space: the preferred landing location in reading Chinese text. Paper presented at the European Conference on Eye Movements, Finland.

Tsai, C. H., \& McConkie, G. W. (1995). The perceptual span in reading Chinese text: A moving window study. Paper presented at the International Conference on the Cognitive Processing of Chinese and Other Asian Languages, Hong Kong.

Tsai, J. L., \& McConkie, G. W. (2003). Where do Chinese readers send their eyes? In J. Hyona, $\mathrm{R}$. Radach \& $\mathrm{H}$. Deubel (Eds.), The mind's eyes: Cognitive and applied aspects of eye movements (pp. 159-176). Amsterdam: North-Holland/Elsevier.

Tsai, J. L., Tzeng, O. J. L., Hung, D. L., \& Yen, N. S. (2000). The perceptual span in reading Chinese passages: A moving window study of eye movement contingent display. Paper presented at the annual meeting of the Chinese Psychology Association, Taipei, Taiwan.

Twain, M. (1997). The awful German language: Appendix D in A tramp abroad. New York: Penguin. (Original work published 1880)

Venezky, R. L. (1999). The American way of spelling: The structure and origins of American English orthography. New York: Guilford.

Wang, L. (2003). Hanyu Ci de Shehui Yuyanxue Yanjiu [A sociolinguistic study on Chinese words]. Beijing: Shangwu Press.

Yang, H. M. (1994). Word perception and eye movements in Chinese reading. Unpublished doctoral dissertation, University of Illinois at Urbana-Champaign.

Yang, H.-M., \& McConkie, G. W. (1994). Eye movement control in Chinese reading. Bulletin of the National TaiNan Teacher's College, 29, 193-229.

Yang, H.-M., \& McConkie, G. W. (1999). Reading Chinese: Some basic eye-movement characteristics. In J. Wang, A. W. Inhoff, \& H.-C. Chen (Eds.), Reading Chinese script: A cognitive analysis (pp. 207-222). Mahwah, NJ: Erlbaum.

Yang, S.-N., \& McConkie, G. W. (2001). Eye movements during reading: a theory of saccade initiation times. Vision Research, 41, 3567-3585.

Yang, S.-N. (2006). An oculomotor-based model of eye movements in reading: The competition/Interaction model. Cognitive Systems Research, 7, 56-69. 


\title{
Appendix A. Reading Materials
}

\begin{tabular}{|c|c|c|c|c|c|c|}
\hline Language & Story & No. of words (char) & Word length & Grade 3 & Grade 5 & Adults \\
\hline \multirow[t]{8}{*}{ English } & BOAT & 60 & 5.35 & $x$ & $X$ & $x$ \\
\hline & MULE & 222 & 5.65 & $x$ & $x$ & $x$ \\
\hline & BASKETBALL & 87 & 5.40 & $x$ & & $x$ \\
\hline & BEES & 264 & 4.90 & $x$ & & $x$ \\
\hline & GOODALL & 385 & 5.65 & $x$ & & $x$ \\
\hline & CELL & 108 & 6.32 & & $X$ & $x$ \\
\hline & MOZART & 249 & 5.07 & & $x$ & $x$ \\
\hline & NEWTON & 429 & 5.96 & & $x$ & $x$ \\
\hline \multirow[t]{8}{*}{ Chinese } & BOAT & $61(83)$ & 1.62 & $X$ & $x$ & $x$ \\
\hline & MULE & $196(250)$ & 1.56 & $x$ & $x$ & $x$ \\
\hline & BASKETBALL & $68(83)$ & 1.48 & $x$ & & $x$ \\
\hline & FISH & $190(234)$ & 1.55 & $x$ & & $x$ \\
\hline & $\mathrm{MAO}$ & $304(426)$ & 1.54 & $x$ & & $x$ \\
\hline & SWALLOW & 129 (162) & 1.67 & & $X$ & $x$ \\
\hline & WU & $248(311)$ & 1.57 & & $X$ & $x$ \\
\hline & QIAN & 349 (534) & 1.70 & & $x$ & $x$ \\
\hline
\end{tabular}

Note: Further information about the reading materials is provided below. All materials are available upon request.

Two stories were chosen to be comparable across languages. The "Power of Boats" (BOAT, see Appendix B) was an expository text on the evolution of boat engines, translated from English to Chinese. "The Foolish Mule" (MULE) was an Aesop fable about a mule who soaked a sack of cotton when crossing a stream in hope to lessen the weight on his back. We used two roughly parallel versions of the same story in both cultures.

The rest of the stories were selected from extracurricular reading books published in China and the United States. They were representative of readings children would naturally encounter everyday. For the English materials, BASKETBALL was a short passage about the origin of the basketball game. BEES and CELL introduced some interesting nature of honeybees and the history of the discovery of cells, respectively. GOODALL, NEWTON, and MOZART were bibliographical stories about two scientists, Jane Goodall and Sir Isaac Newton, and a musician, Wolfgang A. Mozart.

The Chinese materials were similar to the English ones in terms of contents and length. BASKETBALL was also about the history of basketball, although the story differed in details from the English version. FISH and SWALLOW were expository pieces on deep sea fish and the migration of swallows, respectively. There were also three bibliographical stories, as in the English stories. MAO was a story about Yisheng Mao, an architect who is known for his design of many bridges in China. QIAN was about Xuesen Qian, a wellknown Chinese physicist. A story chosen to be parallel to MOZART, WU was a bibliography on Cheng'en Wu, a famous writer in Ming Dynasty who wrote the novel XiYouJi, whose main character, the Money King, is loved by children in China.

\section{Appendix B. A Sample Story: The Power of Boats}

\section{The English version}

Different boats use different kinds of energy for power. In the past, boats usually used sails to harness the power of the wind. Other boats had oars and needed people for power. But many improvements were made over time. The invention of the engine, for example, allowed boats to travel faster and farther. Now some boats use even better motors.

\section{The Chinese version}

\author{
各种不同的船利用不同的动力行驶。古时候, 货船通常得靠帆利用风力前进, 有些 \\ 船需要用人力划桨。后来逐渐改进, 轮船装上了烧煤的蒸汽机, 船就能够驶向更遥 \\ 远的陆地。现在, 一部分船已经有了更好的动力了。
}

